\title{
PROPULSIVE SMALL EXPENDABLE DEPLOYER SYSTEM (ProSEDS) SPACE EXPERIMENT
}

\author{
Mr. Les Johnson \\ Program Development Directorate/PS02 \\ National Aeronautics and Space Administration \\ George C. Marshall Space Flight Center \\ Huntsville, Alabama 35812 \\ les.johnson@msfc.nasa.gov \\ 256-544-0614 (phone) \\ Drs. Robert D. Estes and Enrico Lorenzini \\ Smithsonian Astrophysical Observatory \\ 60 Garden Street, Cambridge, Massachusetts 02138 \\ 617-495-7261 (phone) 617-496-7670 (fax)
}

\author{
Dr. Brian Gilchrist \\ The University of Michigan \\ Space Physics Research Laboratory \\ 2455 Hayward, Ann Arbor, Michigan 48109-2143 \\ 734-763-6230 (phone) 734-764-5137 (fax)
}

\author{
Ms. Judy Ballance \\ Chief Engineers Office/EE61 \\ National Aeronautics and Space Administration \\ George C. Marshall Space Flight Center \\ Huntsville, Alabama 35812 \\ judy.ballance@msfc.nasa.gov \\ 256-544-6042 (phone)
}

\begin{abstract}
The Propulsive Small Expendable Deployer System (ProSEDS) space experiment will demonstrate the use of an electrodynamic tether propulsion system. The flight experiment is a precursor to the more ambitious electrodynamic tether upper stage demonstration mission which will be capable of orbit raising, lowering, and inclination changes-all using electrodynamic thrust. ProSEDS, which is planned to fly in 2000, will use the flight-proven Small Expendable Deployer System (SEDS) to deploy a tether (5-km bare wire plus $15-\mathrm{km}$ Spectra $\left.{ }^{\mathrm{TM}}\right)$ from a Delta II upper stage to achieve $\sim 0.4 \mathrm{~N}$ drag thrust, thus demonstrating deorbit thrust. The experiment will use a predominantly "bare" tether for current collection in lieu of endmass collector and insulated tether approach used on previous missions. ProSEDS will utilize tethergenerated current to provide limited spacecraft power. In addition to the use of this technology to provide orbit transfer of payloads and upper stages from low-Earth orbit (LEO) to higher orbits, it may also be an attractive option for future missions to Jupiter and any other planetary body with a magnetosphere.
\end{abstract}

Copyright (C) 1998 by the American Institute of Aeronautics and Astronautics. Inc. No copyright is asserted in the United States under Title 17. U.S. Code. The U.S. Government has a royalty-free license to exercise all rights under the copyright claimed herein for Governmental Purposes. All other rights are reserved by the copyright owner.

\section{Introduction}

Since the 1960 's, there have been at least 16 tether missions. In the 1990's, several important milestones were reached, including the retrieval of a tether in space, successful deployment of a $20-\mathrm{km}$-long tether in space and operation of an electrodynamic tether with tether current driven in both directions-power and thrust modes. ' A list of known tether missions is shown in Table 1. The ProSEDS mission, to be flown in 2000 , is sponsored by NASA's Advanced Space Transportation Program Office at the George C. Marshall Space Flight Center (MSFC).

\section{ProSEDS Flight Experiment Overview}

A flight experiment to validate the performance of the bare electrodynamic tether in space and demonstrate its capability to produce thrust is planned by NASA for the year 2000. The ProSEDS experiment will be placed into a $400-\mathrm{km}$ circular orbit as a secondary payload from a Delta II launch vehicle (Fig. 1). Once on orbit, the flightproven SEDS will deploy $15 \mathrm{~km}$ of insulating Spectra TM tether attached to an endmass, followed by $5 \mathrm{~km}$ of predominantly bare wire tether (Fig. 2). Upward deployment will set the system to operate in the generator mode, thus producing drag thrust and electrical power. The drag thrust provided by the tether, with an average current of $0.5 \mathrm{~A}$, could deorbit the Delta II upper stage in approximately 17 days, versus its nominal $\geq 6$ months lifetime in a $400-\mathrm{km}$ circular orbit (Fig. 3). Approximately 
Table 1. Known tether flights.

\begin{tabular}{|l|c|l|c|l|}
\hline Name & Date & Orbit & Length & \multicolumn{1}{|c|}{ Comments } \\
\hline Gemini 11 & 1967 & LEO & $30 \mathrm{~m}$ & Spin stable 0.15 rpm \\
Gemini 12 & 1967 & LEO & $30 \mathrm{~m}$ & Local vertical, stable swing \\
H-9M-69 & 1980 & Suborbital & $500 \mathrm{~m}$ & Partial deployment \\
S-520-2 & 1981 & Suborbital & $500 \mathrm{~m}$ & Partial deployment \\
Charge-1 & 1983 & Suborbital & $500 \mathrm{~m}$ & Full deployment \\
Charge-2 & 1984 & Suborbital & $500 \mathrm{~m}$ & Full deployment \\
ECHO-7 & 1988 & Suborbital & $?$ & Magnetic field-aligned \\
Oedipus-A & 1989 & Suborbital & $958 \mathrm{~m}$ & Spin stable 0.7 rpm \\
Charge-2B & 1992 & Suborbital & $500 \mathrm{~m}$ & Full deployment \\
TSS-1 & 1992 & LEO & $<1 \mathrm{~km}$ & Electrodynamic, partial deploy, retrieved \\
SEDS-1 & 1993 & LEO & $20 \mathrm{~km}$ & Downward deploy, swing \& cut \\
PMG & 1993 & LEO & $500 \mathrm{~m}$ & Electrodynamic, upward deploy \\
SEDS-2 & 1994 & LEO & $20 \mathrm{~km}$ & Local vertical stable, downward deploy \\
Oedipus-C & 1995 & Suborbital & $1 \mathrm{~km}$ & Spin stable 0.7 rpm \\
TSS-1R & 1996 & LEO & $19.6 \mathrm{~km}$ & Electrodynamic power generation and \\
& & & science \\
TiPS & 1996 & LEO & $4 \mathrm{~km}$ & Long life tether \\
\hline
\end{tabular}

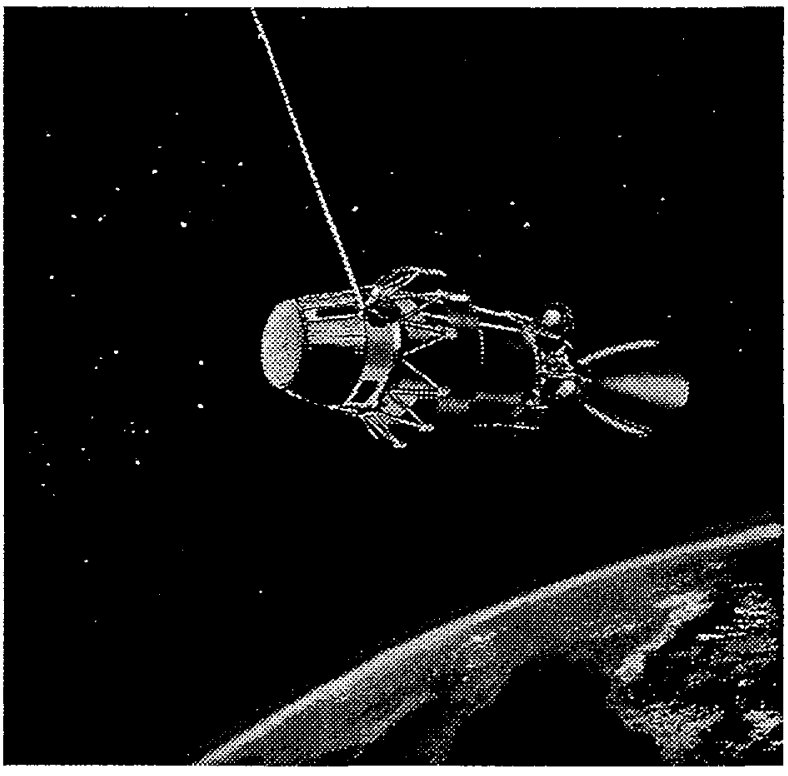

Fig. 1. Artist concept of ProSEDS on Delta II.

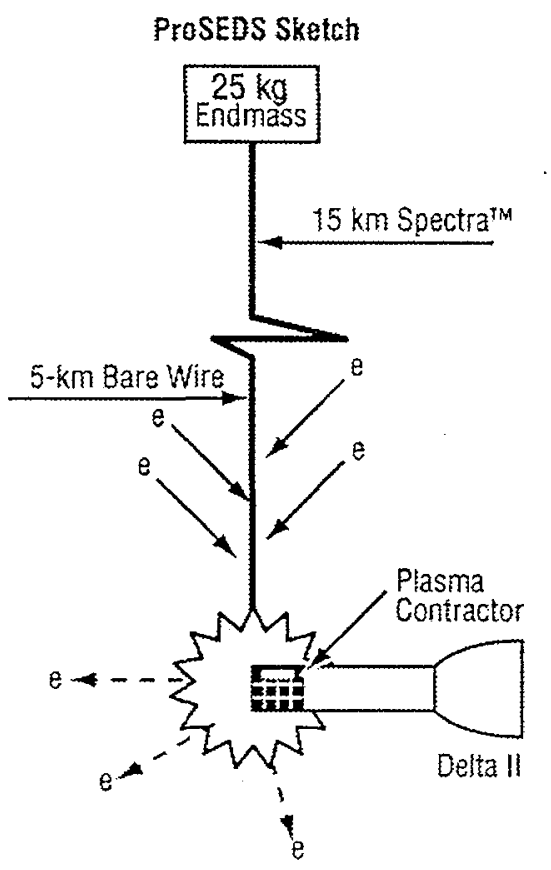

Fig. 2. ProSEDS sketch. 


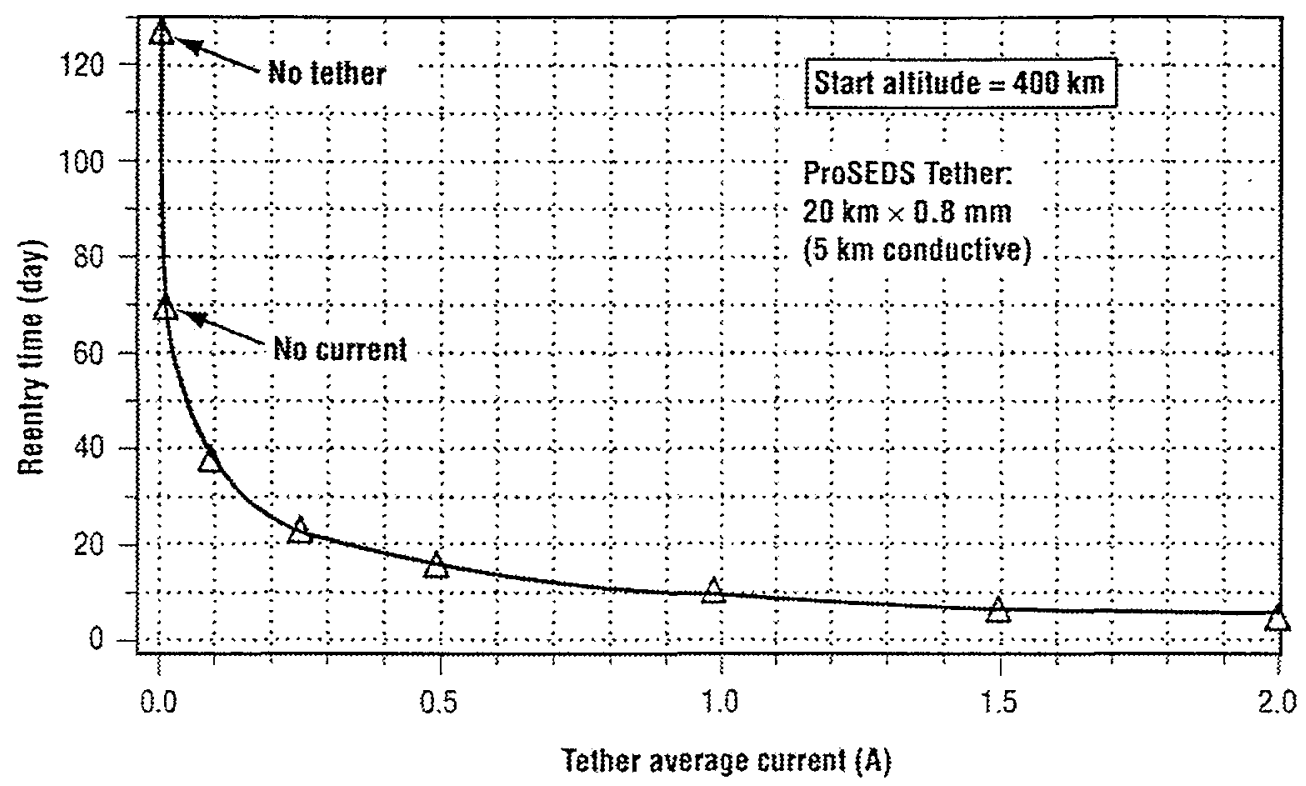

Fig. 3. Predicted performance of ProSEDS propulsive drag thrust. The Delta II reentry time versus tether average current is shown.

$100 \mathrm{~W}$ electrical power will be extracted from the tether to recharge mission batteries and to allow extended measurements of the system's performance. A plasma contactor will be attached to the Delta II to complete the circuit and emit electrons back into space. Performance and diagnostic instruments mounted on the Delta II will be used to correlate the propulsive forces generated by the electrodynamic tether and the existing plasma conditions. These instruments will measure plasma density, temperature, energy, and potential. ProSEDS will be the first tether mission to produce electrodynamic thrust, use a bare wire tether, and recharge mission batteries using tether-generated power.

\section{Electrodynamic Tethers}

The ProSEDS flight experiment will demonstrate electrodynamic propulsion (through drag thrust) in space. From theoretical analyses and preliminary plasma chamber tests, bare tethers appear to be very effective anodes for collecting electrons from the ionosphere and, consequently, attaining high currents with relatively short tether lengths. ${ }^{2}$ A predominantly uninsulated (bare wire) conducting tether, terminated at one end by a plasma contactor, will be used as an electromagnetic thruster. A propulsive force of $\mathbf{F}=I \mathbf{L} \times \mathbf{B}$ is generated on a spacecraft tether system when a current, I, from electrons collected in space plasma, flows down a tether of length, $\mathrm{L}$, due to the emf induced in it by the geomagnetic field, $\mathbf{B}$. Preliminary tests indicate that a thin uninsulated wire could be 40 times more efficient as a collector than previous systems (Fig. 4).

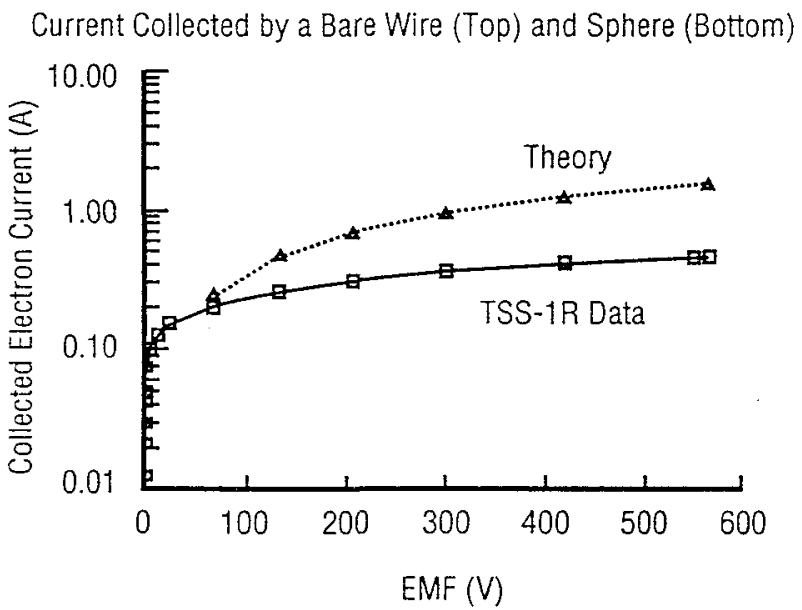

Fig. 4. Current collected by a bare wire versus a sphere in plasma chamber results provided by Jim Sorensen and Nobie Stone/NASA. 


\section{Future Applications for Electrodynamic Tethers}

The main advantage of electrodynamic tethers is that they can be used as propellantless (no resupply required) space propulsion systems. Tethers take advantage of the natural plasma environment and sunlight to provide thrust and power. For example, if solar arrays and an external power supply are used, an emf can be generated in the tether such that current collected from the ionosphere produces thrust rather than drag. This thrust can then be used to raise the orbit of the system or change its inclination-all without propellant or rocket engines. It is envisioned that this type of propulsion could be used on a reusable upper stage to provide a low recurring cost alternative to chemical stages (Fig. 5). The electrodynamic tether upper stage could be used as an orbital tug to move payloads within LEO after insertion. The tug would rendezvous with the payload and launch vehicle, dock/ grapple the payload and maneuver it to a new orbital altitude or inclination within LEO without the use of boost propellant. The tug could then lower its orbit to rendezvous with the next payload and repeat the process. Such a system could conceivably perform several orbital maneuvering assignments without resupply, making it a low recurring cost space asset. The ProSEDS itself could be used operationally to extend the capability of existing launch systems by providing a propellantless system for deorbiting spent stages. The launch service provider need not carry additional fuel for the soon-to-be-required deorbit maneuver, thus allowing all the onboard fuel to be used for increasing the vehicle's performance. Similarly, satellites thus equipped could safely deorbit at their end of life without using precious onboard propellant.

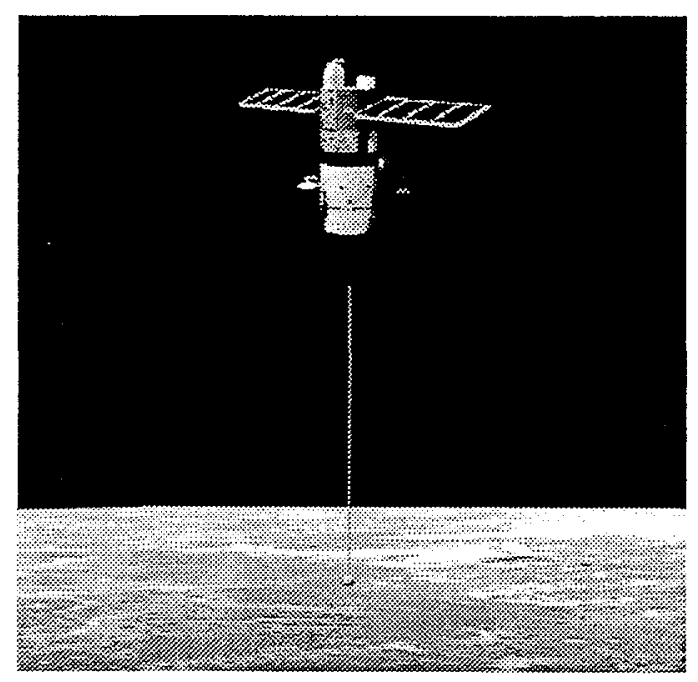

Fig. 5. Artists concept of an electrodynamic tether upper stage.
Both of these applications would help reduce the increasing threat posed by orbital debris. An electrodynamic tether system (Fig. 6) could be used on the International Space Station (ISS) to supply a reboost thrust of $0.5-0.8 \mathrm{~N}$, thus saving up to $6,000 \mathrm{~kg}$ of propellant per year. ${ }^{3}$ The reduction of propellant needed to reboost the ISS equates to a $\$ 2 \mathrm{~B}$ savings over it's 10 -year lifetime. ${ }^{3}$ Other advantages of using the electrodynamic tether on the ISS are that the microgravity environment is maintained and external contaminants are reduced. Yet another use for electrodynamic tethers is the exploration of any planet with a magnetosphere, such as Jupiter. Jupiter's rapid rotation produces a condition where a tether can produce power and raise orbit passively and simultaneously. MSFC is working with the Jet Propulsion Laboratory (JPL) to study the use of electrodynamic tethers for future Jovian missions such as the Jupiter Polar Orbiter (Fig. 7).

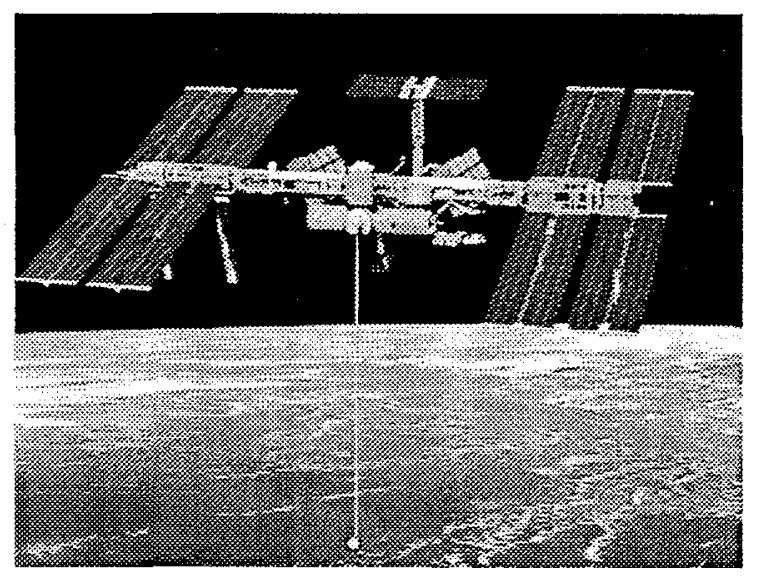

Fig. 6. ISS with electrodynamic tether for reboost.

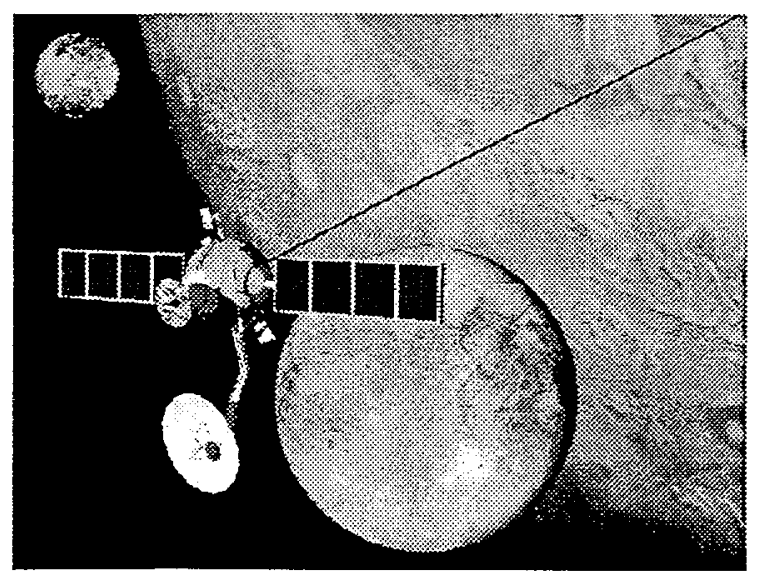

Fig. 7. Jovian electrodynamic tether concept. 


\section{Conclusions}

Tether technology has advanced significantly since its inception over 30 years ago. The recent successes of the SEDS show that tethers are ready to move from experiment and demonstration to application. One of the most promising applications for tethers is space propulsion and transportation. The use of electrodynamic tether propulsion for reusable upper stages, planetary missions, space station, and launch vehicle deorbit applications will soon be demonstrated with the ProSEDS mission. The ProSEDS mission will demonstrate and validate the production of power in space, using a bare wire tether, which produces drag thrust propulsion.

\section{Acknowledgments}

This paper was prepared for the ProSEDS project, which is sponsored by the Advanced Space Transportation Program Office of the National Aeronautics and Space Administration (NASA) located at MSFC. Additional contributions to this paper were provided by Nobie Stone and Jim Sorensen of MSFC.

\section{$\underline{\text { References }}$}

1. Johnson, L., Estes, R., and Lorenzini, E., "Space Transportation Systems Using Tethers," in Sixth Alumni Conference of the International Space University, Houston, TX, NASA Conference Publication 3355, 1997, pp. 89-96.

2. Colombo, G., "Study of Certain Launching Techniques Using Long Tethers," NASA-CR-164060, 1981.

3. Johnson, L., Caroll, J., Estes, R., Gilchrist, B., Lorenzini, E., Martinez-Sanchez, M., Sanmartin, J., and Vas, E., "Electrodynamic Tethers for Reboost of the International Space Station and Spacecraft Propulsion," in 1996 A.IAA Space Programs and Technology Conference, Huntsville, AL, AIAA 96-4250, 1996. 